\title{
THE RESISTIVITY AND HALL EFFECT OF THE AMORPHOUS $\mathrm{Co}_{x} \mathrm{Ni}_{70-x} \mathrm{Fe}_{5} \mathrm{Si}_{15} \mathrm{~B}_{10}$ RIBBONS
}

\author{
K. Perzyńska ${ }^{a}$, M. Biernacka ${ }^{a}$, L. Dobrzyński ${ }^{a, b}$, A. MALinowski ${ }^{a}$, \\ S. TARASENKo ${ }^{a}$ AND P. ZALESKI ${ }^{a}$ \\ anstitute of Physics, University at Białystok, Lipowa 41, 15-424 Białystok, Poland \\ ${ }^{b}$ Sołtan Institute for Nuclear Studies, 05-400 Otwock-Świerk, Poland
}

The influence of structural disorder and magnetism on the resistivity of the amorphous $\mathrm{Co}_{x} \mathrm{Ni}_{70-x} \mathrm{Fe}_{5} \mathrm{Si}_{15} \mathrm{~B}_{10}$ ribbons was investigated. The resistivity vs. alloy composition shows parabolic-like behaviour. In the thermal behaviour, resistivities of the samples show apparent magnetic contribution. They also exhibit minima at temperatures which can be correlated with the Curie temperatures. Above $T \geq 0.5 T_{\mathrm{C}}$ resistivity increases linearly with increasing temperature. This effect is seemingly due to electron-phonon scattering. Obviously, the chemical and positional disorder is responsible for the major part of resistivity.

PACS numbers: $72.15 . \mathrm{Cz}$

\section{Introduction}

We report the results of electric transport measurements on $\mathrm{Co}_{x} \mathrm{Ni}_{70-x} \mathrm{Fe}_{5} \mathrm{Si}_{15} \mathrm{~B}_{10}$ alloys, where nickel was substituted for cobalt. The magnetic properties of these alloys have been studied in detail in [1, 2]. In particular, it has been shown that nickel does not modify the structure of the alloys, therefore its presence leads to extra chemical disorder only. Nickel atoms, however, modify substantially magnetic properties, therefore in principle a systematic study of electric transport phenomena should reveal the influence of both effects.

\section{Experimental}

Ribbons with $x=10,20,30,40,55$, and 70 were made by a rapid quenching on a single copper roller, see [1] for details. The resistivity was measured by a standard four-point DC method from $12 \mathrm{~K}$ to $300 \mathrm{~K}$ in a closed cycle refrigerator. The Hall effect was measured at the same temperature range in the field up to $1.7 \mathrm{~T}$. 


\section{Results and discussion}

A change of resistivities of our samples with temperature is shown in Fig. 1a. The resistivity attains a maximum around $x=40$, see Fig. 1b. However, the temperature variation of the resistivities does not exceed $6.5 \%$ in the temperature range covered by our experiment. In all the ribbons magnetic contributions to the electron transport clearly show up. For samples with $x=10,20$, and 30 , whose Curie temperatures are below $300 \mathrm{~K}$, a change of the slopes of the resistivities vs. temperature appears at the Curie temperatures. The values of the transition temperature, $T_{\mathrm{CR}}$, defined as the point where $\mathrm{d}^{2} \rho / \mathrm{d} T^{2}=0$, are displayed in Table together with the values of the Curie temperatures, $T_{\mathrm{CM}}$, obtained from our earlier magnetometric measurements [1].

\section{TABLE}

Temperatures of resistivity minima $T\left(\rho_{\min }\right)$, transition temperatures obtained from the resistivity $\left(T_{\mathrm{CR}}\right)$ and magnetometric measurements $\left(T_{\mathrm{CM}}\right)$.

\begin{tabular}{c|c|c|c|c|c|c}
\hline \hline$x$ & 10 & 20 & 30 & 40 & 55 & 70 \\
\hline$T\left(\rho_{\min }\right)[\mathrm{K}]$ & 16 & 12 & 14 & 21 & 26 & 40 \\
$T_{\mathrm{CR}}[\mathrm{K}]$ & $40(3)$ & $140(10)$ & $263(3)$ & & & \\
$T_{\mathrm{CM}}[\mathrm{K}]$ & 50 & 150 & 275 & 378 & 540 & 660
\end{tabular}

In contrast to the results of Gomez Sal et al. [3], who studied $\left(\mathrm{Co}_{1-x} \mathrm{Ni}_{x}\right)_{75} \mathrm{Si}_{15} \mathrm{~B}_{10}$ alloys, the temperatures $T_{\mathrm{CR}}$ are systematically slightly smaller than corresponding $T_{\mathrm{CM}}$. Another novel feature is a clear-cut maximum of the resistivity observed in the concentration range in which maximum disorder exists (see Fig. 1b).

Except for the $x=10$ sample in which the temperature variation of resistivity above approximately $0.4 T_{\mathrm{C}}$ is weak, if any, for samples with sufficiently low Curie temperatures the resistivity at $T<0.5 T_{\mathrm{C}}$ varies rather as $T^{3 / 2}$ than $T^{2}$. It suggests that incoherent spin-wave scattering gives a dominant contribution in this region of temperature. According to the results of Babic et al. [4] the $T^{3 / 2}$ dependence should hold for temperatures up to $0.5 T_{\mathrm{C}}$ and $T^{2}$ or linear for higher ones. In our case, a linear variation of the resistivity with temperature was found at $T \geq 0.5 T_{\mathrm{C}}$ for the samples with low $T_{\mathrm{C}}$ values which is in excellent agreement with observation of Barandiaran et al. [5], Kaul et al. [6], and Das et al. [7] on similar alloys. But we note that temperature behaviour of the resistivity is directly connected with Co-concentration. For $x \leq 0.55$ a $T^{3 / 2}$-dependence is found. This can be explained by the incoherent electron-magnon scattering predicted by theory of Richter et al. [8] and observed in Ref. [4]. At higher Co content a clear-cut $T^{2}$-dependence at low temperature is observed, which can be due either to magnetic (coherent spin-wave scattering) or to the structural contribution, see Refs. $[6,7]$.

All our samples exhibit resistance minima at temperatures increasing with Co concentration, i.e. also with increasing Curie temperature (see Table). This 

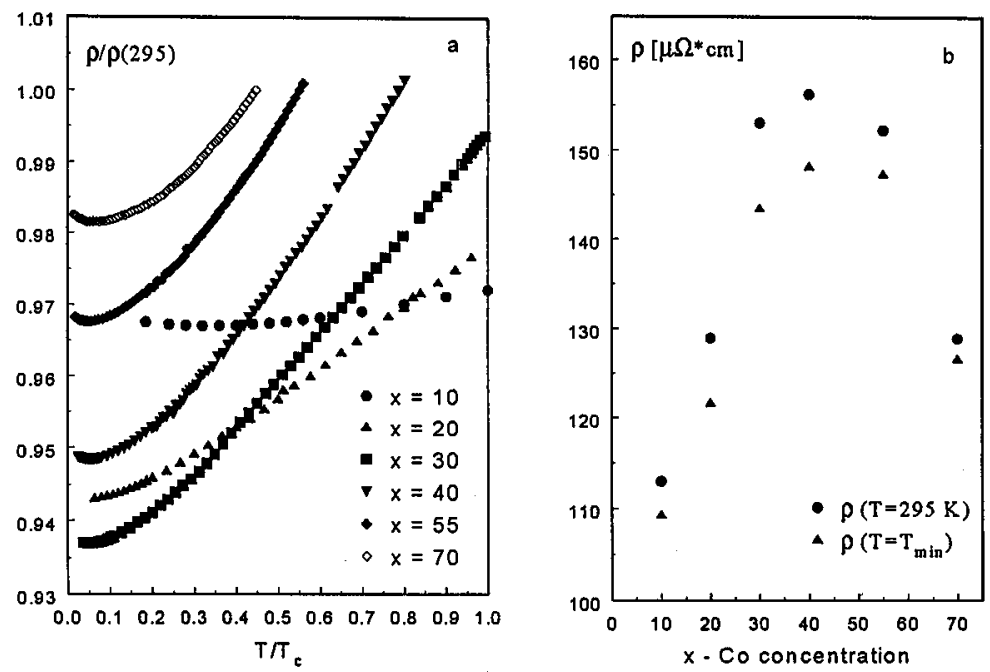

Fig. 1. Normalised resistivity as a function of normalised temperature of $\mathrm{Co}_{x} \mathrm{Ni}_{70-x} \mathrm{Fe}_{5} \mathrm{Si}_{15} \mathrm{~B}_{10}$ alloys (a). The resistivity as a function of Co-concentration at $T=T_{\min }$ and $T=295 \mathrm{~K}(\mathrm{~b})$.
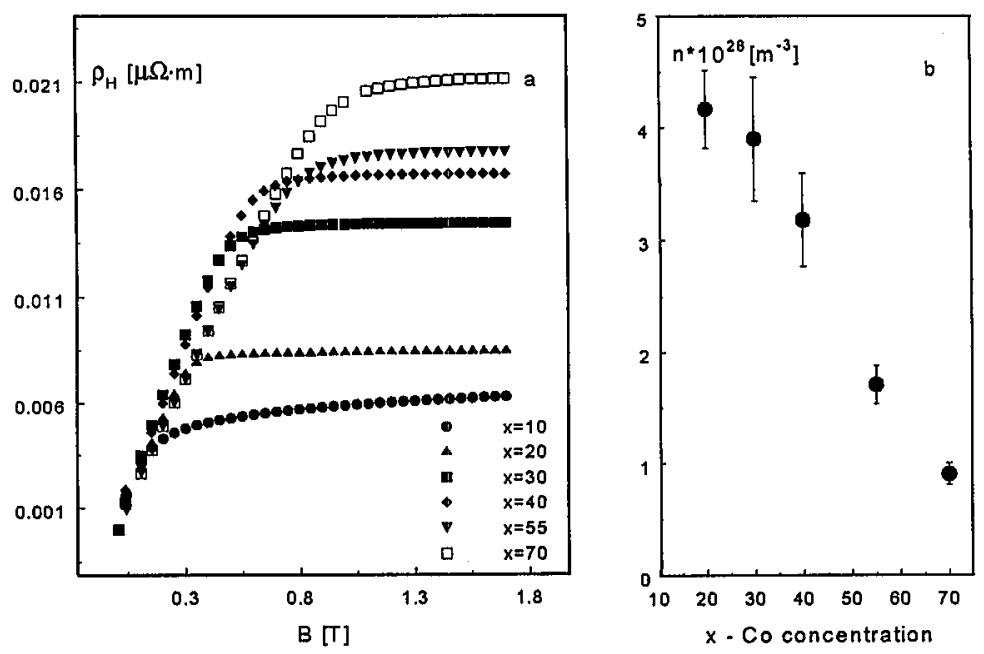

Fig. 2. The Hall resistivity vs. applied magnetic induction (a). The concentration of current carriers as function of Co-concentration (b). $\mathrm{Co}_{x} \mathrm{Ni}_{70-x} \mathrm{Fe}_{5} \mathrm{Si}_{15} \mathrm{~B}_{10}$ in $T=12 \mathrm{~K}$.

fact suggests that the effect may be of magnetic origin. The presence of spin-glass like states found in the samples with $x<30$, see [1], can lead to extra scattering mechanism and to the increase in resistivity at the lowest temperatures. This would explain why the temperature of the resistivity minimum for $x=10$ is higher than for the sample with $x=20$.

We also measured the Hall resistivity $\rho_{\mathrm{H}}$ in the temperature range from $12 \mathrm{~K}$ to $295 \mathrm{~K}$. All the ribbons show ferromagnetic behaviour at $T=12 \mathrm{~K}$ (see Fig. $2 \mathrm{a}$ ). 
The magnetic moments per transition metal atom at $12 \mathrm{~K}$, estimated from both Hall effect and magnetisation measurements (on vibrating sample magnetometer, VSM) agree very well with each other. The observed positive values of $R_{H}$ can be explained within the framework of " $s-d$ hybridisation" model [9], which shows that at the Fermi energy the electron group velocity may be negative and hence the Hall coefficient - positive.

Concentrations of electrons $n$ were estimated from the ordinary Hall coefficient $R_{0}=1 / n e$ [9]. The obtained values depend strongly on temperature and alloy composition. The $n(x)$ dependence obtained at $12 \mathrm{~K}$ is shown in Fig. $2 \mathrm{~b}$.

If the current carriers concentration is transformed to the number of conduction electrons per molecule, a change of this number with $x$ is in a good agreement with the results of the Compton scattering [10]. It seems that it is for the first time when such two different experiments could be confronted with each other. It is also shown that in spite of the chemical complexity, the alloys of interest behave as pseudobinary alloys and the resistivity follows the Nordheim rule. The resistivity minima are shown to be largely of magnetic origin in contrast to the results published in Ref. [11].

\section{References}

[1] L. Dobrzyński, K. Szymański, J. Waliszewski, A. Malinowski, A. Wiśniewski, M. Baran, J. Latuszkiewicz, J. Magn. Magn. Mater. 88, 23 (1990).

[2] L. Dobrzyński, M.Th. Rekveldt, J. Magn. Magn. Mater. 94, 153 (1991).

[3] J.C. Gomez Sal, J.F. Rodriguez, L.B. Fernandez, J.M. Barandiaran, F. Plazaola, in: Proc. Symp. on Magnetic Properties of Amorphous Metals, Ed. A. Hernando, North-Holland, Amsterdam 1987, p. 185.

[4] E. Babić, M. Očko, Ž. Marohnič, A.S. Schaafsma, I. Vincze, J. Phys. Colloque C8, supplement au no 841 , C8-473 (1980).

[5] J.M. Barandiaran, J.C. Gomez-Sal, J.F. Rodrigez, R.J. Lopez Sanchez, O.V. Nielsen, Phys. Status Solidi A 99, 243 (1987).

[6] S.N. Kaul, W. Kettler, M. Rosenberg, Phys. Rev. B 33, 4987 (1986).

[7] A. Das, A.K. Majumdar, Phys. Rev. B 43, 6042 (1991).

[8] R. Richter, M. Wolf, F. Goedsche, Phys. Status Solidi B 95, 473 (1979).

[9] J.S. Dugdale, The Electrical Properties of Disordered Metals, University Press, Cambridge 1995, p. 240.

[10] E. Żukowski, L. Dobrzyński, M.J. Cooper, D.N. Timms, R.S. Holt, J. Latuszkiewicz, J. Phys., Condens. Matter 2, 6315 (1990).

[11] R.W. Cochrane, R. Harris, J.O. Strom-Olson, M.J. Zuckermann, Phys. Rev. Lett. 35, 676 (1975). 